\title{
A decision support model for cost-effectiveness of radical prostatectomy in localized prostate cancer
}

\author{
Johan Lyth, Swen-Olof Andersson, Ove Andrén, Jan-Erik Johansson, \\ Per Carlsson and Nosrat Shahsavar
}

\section{Linköping University Post Print}

N.B.: When citing this work, cite the original article.

Original Publication:

Johan Lyth, Swen-Olof Andersson, Ove Andrén, Jan-Erik Johansson, Per Carlsson and Nosrat Shahsavar, A decision support model for cost-effectiveness of radical prostatectomy in localized prostate cancer, 2012, Scandinavian Journal of Urology and Nephrology, (46), 1, $19-25$.

http://dx.doi.org/10.3109/00365599.2011.615759

Copyright: Informa Healthcare

http://informahealthcare.com/

Postprint available at: Linköping University Electronic Press

http://urn.kb.se/resolve?urn=urn:nbn:se:liu:diva-70933 


\section{A Decision Support Model for Cost Effectiveness of Radical}

\section{Prostatectomy in Localized Prostate Cancer}

Johan Lyth, ${ }^{\mathrm{a}, \mathrm{b}}$ Swen-Olof Andersson, ${ }^{\mathrm{c}}$ Ove Andrén, ${ }^{\mathrm{c}}$ Jan-Erik Johansson, ${ }^{\mathrm{c}}$ Per Carlsson, ${ }^{\mathrm{d}}$ and Nosrat Shahsavar ${ }^{\mathrm{a}, \mathrm{b}}$
a) Department of Biomedical Engineering, Division of Medical Informatics, Linköping University, Linköping, Sweden
b) Regional Cancer Center Southeast, Linköping University Hospital, Linköping, Sweden
c) Department of Urology, Örebro University Hospital, Örebro, Sweden
d) Center for Medical Technology Assessment, Linköping University, Linköping, Sweden

Corresponding author:

Johan Lyth

Regional Cancer Center Southeast

University Hospital

SE-581 85 Linköping, Sweden

Tel: +46101033488

johan.lyth@liu.se

Word count Abstract: 246

Word count Text: 3013 


\begin{abstract}
Objective: This study aims to develop a probabilistic decision support model to calculate the lifetime incremental cost-effectiveness ratio (ICER) between radical prostatectomy and watchful waiting for different patient groups.

Material and Methods: A randomized trial (SPCG-4) provided most data for this study. Data on survival, costs, and quality of life are inputs in a decision analysis, and a decision support model has been developed. The model can generate cost-effectiveness information on subgroups of patients having different characteristics.
\end{abstract}

Results: Age is the most important independent factor explaining cost effectiveness. The costeffectiveness value varies from 21026 Swedish kronor (SEK) to 858703 SEK for those aged 65 to 75 years, depending on Gleason scores and prostate specific antigen (PSA) values.

Information from the decision support model can support decision makers in judging whether or not radical prostatectomy (RP) should be used to treat a specific patient group.

Conclusions: The cost-effectiveness ratio for RP varies with age, Gleason scores, and PSA values. Assuming a threshold value of 200000 SEK per QALY gained, for patients aged $<=70$ years the treatment is always cost effective, except at age 70, Gleason 0-4, and PSA $<=10$. Using the same threshold value at age 75, Gleason 7-9 (regardless of PSA) and Gleason 5-6 (with PSA >20) are cost effective. Hence, RP is not perceived to be cost effective in males aged 75 years having low Gleason and low PSA. Higher threshold values for patients with clinically localized prostate cancer could be discussed.

Key words: decision support, cost effectiveness, randomized trial, prostate cancer, watchful waiting, radical prostatectomy 


\section{Introduction}

The widespread use of prostate specific antigen (PSA) testing has led to a dramatic increase in detection of localized prostate cancer in most Western countries $(1,2)$. Different treatment options are available to these patients in early stages. The most common option is radical prostatectomy (RP), but patients with newly diagnosed, localized, prostate cancer can also be managed in an expectative setting, but where clinicians expect to cure patients with radical surgery or radiotherapy if the tumor shows signs of progression. In recent years, active surveillance has become more common (3). The lack of convincing scientific evidence on effectiveness still makes the choice of treatment strategy for localized prostate cancer controversial in many patient groups. A decision support model based on a decision analysis approach can offer helpful guidance in determining which treatment to choose for different patient groups (4). Apart from appraising effectiveness in terms of survival, in most policymaking situations it is relevant to consider health-related quality of life (HRQoL) and cost effectiveness. A decision support model encompassing these factors should provide information for decision makers, providing them support in setting priorities when creating treatment guidelines. To handle uncertainty within the given parameters, a probabilistic sensitivity analysis is recommended (5).

Some studies on localized prostate cancer have attempted to handle uncertainty by using a decision analytic approach, but with varying quality of data (6-8). Although a randomized clinical trial (RCT) on the lifetime cost effectiveness of RP has never been reported, this type of assessment should be of substantial importance in developing an evidence-based policy for managing localized prostate cancer. The randomized trial, Scandinavian Prostatic Cancer Group Study Number 4, SPCG-4 (www.roc.se) compares watchful waiting (WW) with RP and investigates specific outcomes, e.g. survival, HRQoL, and costs. The results (presented in four papers) show that compared to watchful waiting, RP yields in gain in survival time for 
some patient groups, depending on the patient's age, Gleason score, and PSA value (9-12). According to these reports, patients treated with RP have similar or higher HRQoL than patients treated with WW $(13,14)$. Andersson et al studied the long-term cost of the two treatments and reported a lower cost for the WW group compared to the RP group (15). Slightly longer survival is gained at a higher cost. Probabilistic reasoning based on the data from these studies can be used to support decision makers in judging whether the extra cost per health-year gained is reasonable.

This study aims to develop a probabilistic decision support model to calculate the lifetime incremental cost-effectiveness ratio (ICER) between RP and WW. Since risk at baseline is a potentially important predictor of prostate cancer progression, the decision support model should also calculate the cost effectiveness for subgroups with different risk profiles. In this study, we combine survival, HRQoL, and cost data from the SPCG-4 trial (including full costeffectiveness analysis) to provide guidance concerning which treatment to use based on different patient characteristics.

\section{Material and Methods}

Our study uses a decision support method based on a semi-Markov model (Figure 1) to handle all of the parameters and to extrapolate data for calculating lifetime mean costs and the number of quality-adjusted life-years (QALYs) per patient for the two treatment strategies. We took a probabilistic approach to include uncertainty in the model. We calculated costs and number of QALYs for each year that a patient spends in each state in the semi-Markov model, allowing us to handle probabilities that vary with time spent in a specific state. A structured analysis model of prostate cancer interventions (Figure 1) was first published in 1993 (16). We have modified the Markov model to use symptomatic disease instead of hormonally controlled metastasis and to use refractory disease instead of refractory metastasis. An 
assumption in the model is that patients cannot die of prostate cancer unless they have developed a refractory disease.

The analysis takes the perspective of the Swedish healthcare system, and costs are expressed in Swedish currency (SEK) at 2007 prices. Health outcomes were estimated in quality-adjusted life-years (QALYs). Both costs and QALYs were discounted by $3.5 \%$ per year.

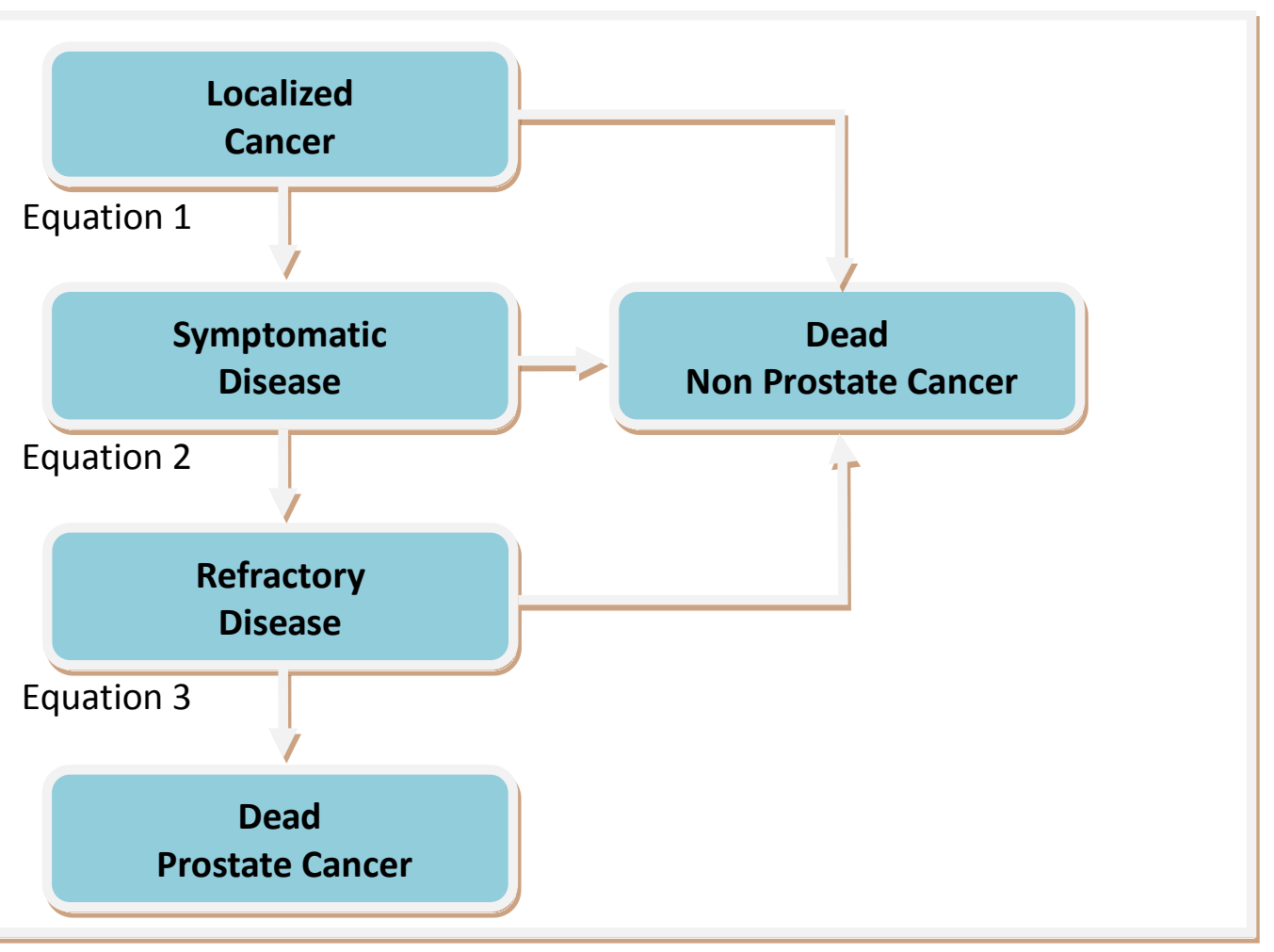

Figure 1. Markov model of prostate cancer

The transition probabilities (risks) of moving from localized prostate cancer to symptomatic disease, from symptomatic disease to refractory disease, and from refractory disease to death in prostate cancer were taken from individual-patient data from SPCG-4. Costs and health-related quality of life were also estimated from individual-patient data from SPCG-4, but costs were based on a subsample of SPCG-4. The probability of dying from causes other than prostate cancer was estimated from Swedish standard life-tables. 


\section{Data sources}

We used data from the 695 patients enrolled in SPCG-4 between October 1989 and February 1999 (9-11). Eligible patients were aged <75 years, life expectancy was >10 years, and no other malignancy was present. Tumors had to be newly diagnosed and localized to the prostate, and the PSA value had to be $<50 \mathrm{ng} / \mathrm{mL}$. The patients were randomized to either early surgery (RP) or a conservative strategy (WW). The surgery started with pelvic lymphadenectomy, and if the histopathology evaluations of the removed lymph nodes were negative, RP was performed. In the WW group, no immediate treatment was performed, but transurethral resection was first-line treatment for symptom relief due to infravesical obstructions.

\section{Transition probabilities (risks)}

All statistical analyses included previously identified baseline risk factors for prostate cancer progression and randomized treatment as separate covariates, namely Gleason $(2-4,5-$ 6, 7, 8-9, missing), PSA (<4ng/mL, 4-6.9 ng/mL, 7-10ng/mL, 10.1-20ng/mL, $>20 \mathrm{ng} / \mathrm{mL}$ or missing), age, and treatment (RP, WW) (11).

The general approach involves testing all covariates with both an exponential distribution and a Weibull distribution, where the exponential distribution handles risk as constant while the Weibull distribution is used when the risk is time-dependent (17). Log-likelihood ratio tests (18) were used to find the distribution that best fit the dataset and to select which explanatory variables to use. By using this method, some of the explanatory variables in the estimation of event rates were retained regardless of statistical significance (Figure 1). A covariance matrix was calculated between the parameters, and Cholesky decomposition (19) was used to account for dependence between parameters. 
In Figure 1, equation 1 represents the annual risk of moving from localized prostate cancer to the symptomatic disease state. The best model was a Weibull distributed model with the following explanatory variables; intercept, Gleason (categorical), PSA (categorical), age (continuous), treatment (categorical), and interaction between age and treatment. Equation 2 represents the annual risk of moving from the symptomatic disease state to the refractory disease state. The best model was an exponential, distributed model using only the intercept as the explanatory variable. Equation 3 represents the annual risk of moving from the refractory disease state to mortality from prostate cancer. The best model was a Weibull distribution model that used intercept and treatment (categorical) as explanatory variables.

The mortality risk from other causes was estimated using Swedish age-specific life-tables for men, adjusted to exclude prostate-cancer-specific mortality (www.scb.se).

\section{Costs}

Data related to the resources used for prostate cancer were collected retrospectively in SPCG-4 by reading up to 17 years of patient records, as reported in detail elsewhere (15).

We used generalized linear model (GLM) to estimate the annual mean cost per state (20). The GLM method was used with Gamma distribution and a Log-link to obtain the best cost for each disease state (Figure 1). Before implementing the GLM, replicated observations were used to estimate the mean cost per patient and year. The Akaikes Information Criterion (AIC) was used in choosing which explanatory variables to include in the analysis (21). Again, Cholesky decomposition was used in accounting for dependence between variables.

\section{Health-related quality of life}

Health-related quality-of-life (HRQoL) data were collected for Swedish patients who were still alive on several occasions in the SPCG-4 study when a 77-item questionnaire with a 
visual analogue scale (VAS) was used to measure HRQoL. The patients estimated their HRQoL at several times and in different disease states. Replicate observations per patient were used when calculating the HRQoL estimates for the three disease states in the model.

To estimate QALYs for each treatment strategy, the patients' time in each health state was multiplied by quality-adjustment weights (utilities) on a scale where 0 represents death and 1 represents full health (taken from the VAS score). We used a two-step procedure to obtain the mean values for the three different health states and to stratify treatment values. First an individual mean value is calculated for each patient for a specific state and treatment. Then a state- and treatment-specific mean value is calculated based on the individual mean values. All data were analyzed using PASW Statistics 18.

\section{Cost effectiveness}

The incremental cost-effectiveness ratio (ICER) is used to measure cost effectiveness. Cost-effectiveness measures the additional sum one must pay to gain an extra QALY, i.e. one year with full health, and is calculated by dividing the difference in mean cost by the mean QALY between the two treatment strategies. We used age, Gleason score, and PSA value in stratification since these are the most important factors in estimating the ICER.

\section{Results}

The decision support model developed in this study is based on the reasoning that resulted from probabilistic calculations of data from SPCG-4. Costs and QALYs are calculated from each disease state (Figure 1) for the two different treatment strategies. These measures are then summarized and discounted, and the mean values are calculated from each probabilistic simulation to obtain the cost-effectiveness value. 


\section{Cost effectiveness}

Table I shows the difference in cost between the two strategies, the difference in QALY, and the cost-effectiveness ratio or ICER. Also presented is the probability that RP is cost effective at certain threshold values of ICER. The most important factors in estimating ICER, patient's age, Gleason score, and PSA are shown for different values. Age is the most important independent factor explaining the size of the ICER; the higher the age, the higher the cost-effectiveness ratio. In other words, it costs more to gain an extra QALY in elderly age groups. Gleason and PSA are important factors in the ICER, especially at older ages. The value of the ICER varies from 21026 SEK to 858703 SEK (Table I) and the ICER is quite low even at 70 years of age, regardless of Gleason score. Only the group with Gleason 0-4 and PSA $<=10$ generates an ICER above 200000 SEK.

At 80 years of age (not shown in Table I) most of the ICERs exceed 500000 SEK, with the exception of Gleason 7-9 and PSA >10, which generate ICERs below 500000 SEK (range 248210 SEK to 448451 SEK). The reason for high ICER values at 80 years of age and above is that the remaining life expectancy is short, and the potential benefits of RP are not fully achieved.

The probability of RP being cost effective at certain values is $100 \%$ regardless of Gleason, even at the low value of 100000 SEK at 55 to 60 years of age (not shown in Table I). The probability at age 70 and Gleason $0-4$, and at age 75 and Gleason $8-9$, is similar at a threshold value of 200000 SEK. 
Table I. Cost-effectiveness results by different patient groups

\begin{tabular}{|c|c|c|c|c|c|c|c|c|c|c|c|c|c|}
\hline & & \multicolumn{12}{|c|}{ Gleason } \\
\hline & & \multicolumn{3}{|c|}{$0-4$} & \multicolumn{3}{|c|}{$5-6$} & \multicolumn{3}{|c|}{7} & \multicolumn{3}{|c|}{$8-9$} \\
\hline & & \multicolumn{3}{|c|}{ PSA } & \multicolumn{3}{|c|}{ PSA } & \multicolumn{3}{|c|}{ PSA } & \multicolumn{3}{|c|}{ PSA } \\
\hline & & $<=10$ & $11-20$ & $>20$ & $<=10$ & $11-20$ & $>20$ & $<=10$ & $11-20$ & $>20$ & $<=10$ & $11-20$ & $>20$ \\
\hline & Incremental cost (SEK) & 40116 & 29805 & 22955 & 49784 & 46043 & 44525 & 52501 & 53726 & 58714 & 67391 & 70108 & 74314 \\
\hline & Incremental QALY & 0.57 & 0.85 & 1.09 & 0.86 & 1.14 & 1.37 & 1.25 & 1.44 & 1.52 & 1.42 & 1.51 & 1.50 \\
\hline & ICER (SEK) & 70766 & 34873 & 21026 & 58045 & 40444 & 32511 & 41834 & 37397 & 38606 & 47482 & 46527 & 49643 \\
\hline \multicolumn{14}{|c|}{$\stackrel{\infty}{\curvearrowright}{ }^{\infty}$ Probability RP is cost effective at: } \\
\hline$\widehat{8}$ & 100000 SEK & 0.64 & 0.87 & 0.96 & 0.79 & 0.94 & 0.98 & 0.96 & 0.99 & 0.99 & 0.96 & 0.97 & 0.97 \\
\hline$\Phi$ & 200000 SEK & 0.79 & 0.93 & 0.98 & 0.92 & 0.98 & 1.00 & 0.99 & 1.00 & 1.00 & 0.99 & 1.00 & 1.00 \\
\hline & 300000 SEK & 0.84 & 0.94 & 0.99 & 0.94 & 0.98 & 1.00 & 1.00 & 1.00 & 1.00 & 1.00 & 1.00 & 1.00 \\
\hline & 400000 SEK & 0.85 & 0.95 & 0.99 & 0.95 & 0.99 & 1.00 & 1.00 & 1.00 & 1.00 & 1.00 & 1.00 & 1.00 \\
\hline & 500000 SEK & 0.87 & 0.96 & 0.99 & 0.95 & 0.99 & 1.00 & 1.00 & 1.00 & 1.00 & 1.00 & 1.00 & 1.00 \\
\hline & & \multicolumn{12}{|c|}{ Gleason } \\
\hline & & \multicolumn{3}{|c|}{$0-4$} & \multirow{2}{*}{\multicolumn{3}{|c|}{$5-6$}} & \multicolumn{3}{|c|}{7} & \multicolumn{3}{|c|}{$8-9$} \\
\hline & & \multicolumn{3}{|c|}{ PSA } & & & & \multicolumn{3}{|c|}{ PSA } & \multicolumn{3}{|c|}{ PSA } \\
\hline & & $<=10$ & $11-20$ & $>20$ & $<=10$ & $11-20$ & $>20$ & $<=10$ & $11-20$ & $>20$ & $<=10$ & $11-20$ & $>20$ \\
\hline & Incremental cost (SEK) & 59160 & 53624 & 47965 & 63834 & 61211 & 59947 & 65085 & 65536 & 66798 & 73820 & 75212 & 76986 \\
\hline & Incremental QALY & 0.25 & 0.41 & 0.56 & 0.42 & 0.60 & 0.77 & 0.65 & 0.80 & 0.96 & 0.83 & 0.95 & 1.02 \\
\hline & ICER (SEK) & 232409 & 129799 & 85681 & 150274 & 101957 & 78074 & 99760 & 82417 & 69887 & 89244 & 79520 & 75302 \\
\hline \multicolumn{14}{|c|}{$\stackrel{\infty}{\mathscr{N}}$} \\
\hline i & 100000 SEK & 0.19 & 0.38 & 0.57 & 0.30 & 0.49 & 0.67 & 0.52 & 0.66 & 0.80 & 0.60 & 0.70 & 0.76 \\
\hline 8 & 200000 SEK & 0.49 & 0.65 & 0.80 & 0.62 & 0.81 & 0.91 & 0.82 & 0.90 & 0.96 & 0.90 & 0.96 & 0.96 \\
\hline & 300000 SEK & 0.59 & 0.74 & 0.86 & 0.74 & 0.87 & 0.95 & 0.89 & 0.95 & 0.98 & 0.95 & 0.98 & 0.98 \\
\hline & 400000 SEK & 0.64 & 0.78 & 0.88 & 0.79 & 0.89 & 0.96 & 0.91 & 0.97 & 0.99 & 0.97 & 0.99 & 0.99 \\
\hline & $500000 \mathrm{SEK}$ & 0.67 & 0.81 & 0.89 & 0.82 & 0.90 & 0.97 & 0.93 & 0.97 & 0.99 & 0.97 & 1.00 & 0.99 \\
\hline & & \multicolumn{12}{|c|}{ Gleason } \\
\hline & & \multirow{2}{*}{\multicolumn{3}{|c|}{$\frac{0-4}{P S A}$}} & \multirow{2}{*}{\multicolumn{3}{|c|}{$\begin{array}{l}5-6 \\
\text { PSA }\end{array}$}} & & 7 & & & $8-9$ & \\
\hline & & & & & & & & & PSA & & & PSA & \\
\hline & & $<=10$ & $11-20$ & $>20$ & $<=10$ & $11-20$ & $>20$ & $<=10$ & $11-20$ & $>20$ & $<=10$ & $11-20$ & $>20$ \\
\hline & Incremental cost (SEK) & 70074 & 67128 & 65002 & 72439 & 71238 & 70409 & 72625 & 72713 & 72905 & 76538 & 77452 & 78164 \\
\hline & Incremental QALY & 0.08 & 0.16 & 0.25 & 0.15 & 0.25 & 0.36 & 0.30 & 0.40 & 0.52 & 0.42 & 0.52 & 0.61 \\
\hline 0 & ICER (SEK) & 858703 & 427571 & 261696 & 472327 & 285780 & 195824 & 244774 & 180284 & 140420 & 181970 & 150296 & 127529 \\
\hline 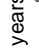 & *Probability RP is cost effective at: & & & & & & & & & & & & \\
\hline$\hat{\kappa}$ & 100000 SEK & 0.02 & 0.05 & 0.11 & 0.02 & 0.06 & 0.13 & 0.07 & 0.14 & 0.26 & 0.11 & 0.16 & 0.28 \\
\hline 8 & 200000 SEK & 0.19 & 0.27 & 0.41 & 0.24 & 0.35 & 0.53 & 0.40 & 0.56 & 0.70 & 0.57 & 0.69 & 0.79 \\
\hline \& & 300000 SEK & 0.33 & 0.41 & 0.55 & 0.41 & 0.53 & 0.68 & 0.57 & 0.73 & 0.82 & 0.73 & 0.85 & 0.90 \\
\hline & 400000 SEK & 0.39 & 0.50 & 0.63 & 0.49 & 0.61 & 0.75 & 0.66 & 0.79 & 0.88 & 0.80 & 0.89 & 0.92 \\
\hline & 500000 SEK & 0.44 & 0.55 & 0.68 & 0.54 & 0.66 & 0.79 & 0.72 & 0.83 & 0.90 & 0.84 & 0.92 & 0.93 \\
\hline
\end{tabular}

Figure 2 presents an example of decision support implementation using probabilistic sensitivity analysis based on calculations using equations 1 to 3 in Figure 1 . In steps 1 to 3 , the characteristics (i.e. age, Gleason score, and PSA value) of the patient group of interest can be entered. The program then runs the probabilistic reasoning module to calculate the cost and the QALY based on survival and HRQoL data for RP and WW. The cost column shows the costs of the two treatment strategies and the difference between them. The QALY column shows the QALY generated by the two strategies and the difference between them. The last result shown is the ICER, where the cost of gaining a QALY is calculated for RP versus WW. In this example, the RP strategy costs an additional 46043 SEK, but generates 1.14 QALY more than the WW strategy. Hence, the ICER is $(46043$ / 1.14) $=40444$ SEK. Decision 
makers can use this information to support judgments on whether or not a specific patient group should be treated with RP, their threshold values, and other priority setting.

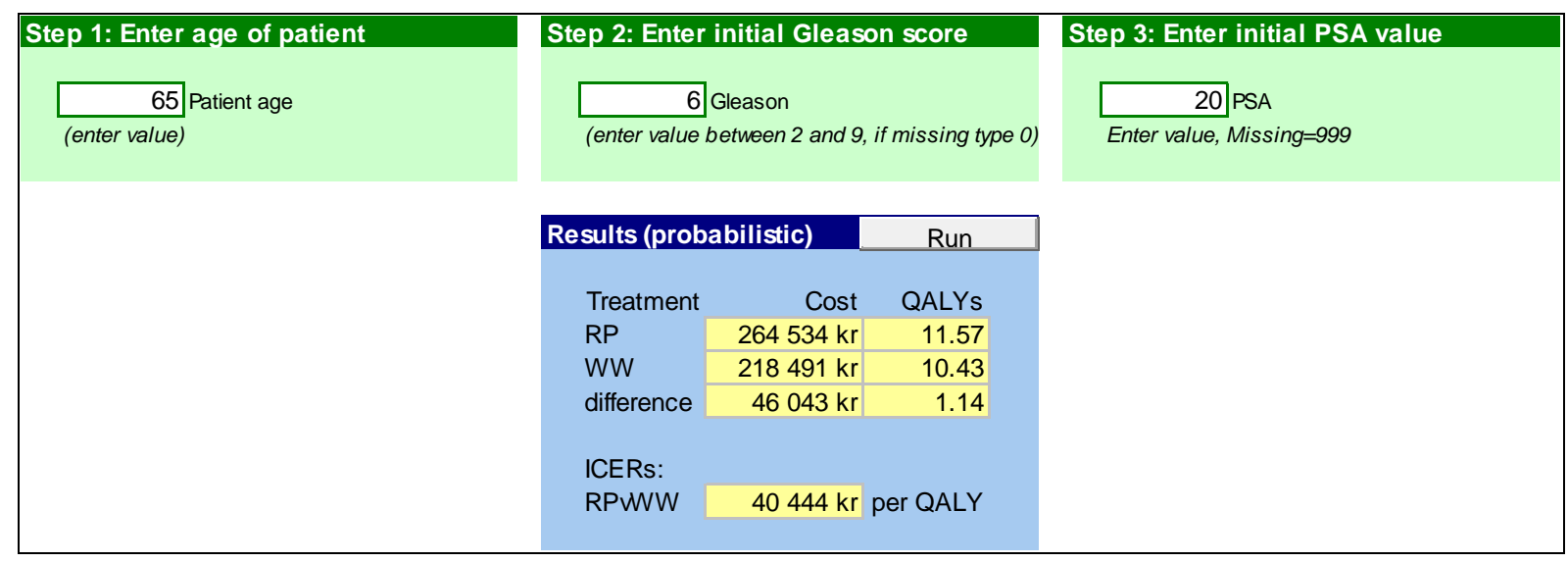

Figure 2. Application of the decision support model. RP=Radical prostatectomy;

WW=Watchful waiting; ICER=Incremental cost-effectiveness ratio; QALY=Quality-adjusted life-years

\section{Discussion}

\section{Decision analysis}

Cost-effectiveness ratios of combinations of interventions and defined patient groups, together with defined threshold values, could be used in making policy decisions (22). Is RP cost effective in relation to $\mathrm{WW}$, and should the procedure be recommended for routine use? Sweden has not defined the thresholds of cost effectiveness for different health conditions. Rather, severity and other relevant aspects are considered in determining when a treatment option should be recommended for routine use in health services. The National Institute for Health and Clinical Excellence in the UK uses a threshold of around 20000 to 30000 pounds sterling (GBP) per QALY gained. Therefore, we have developed a decision support model and calculated the probability of RP being cost effective at several cut-off values of the ICER, i.e. 
levels of willingness-to-pay for health (Table I, Figure 2).

We evaluated uncertainty in cost effectiveness by using probabilistic sensitivity analysis where inputs into the analysis are defined as probability distributions reflecting the precision with which they are estimated (23). To illustrate the variability in costs and QALYs, we estimated the cost effectiveness of RP by using data from different patient groups. These groups are based on different ages, Gleason and PSA values since these are the best prognostic factors concerning prostate cancer mortality. Heredity and unhealthy lifestyle are other possible risk factors for mortality in prostate cancer patients. For more precise estimates, the PSA ratio could also be a good complement to PSA. Unfortunately, these data were not collected in the SPCG-4 trial, but may be considered in future versions of the model.

Decision makers can use the results as a base when constructing treatment guidelines for patient groups with localized prostate cancer. However, they should use their priorities and ICER threshold values as a basis for determining which groups are cost effective.

Table I presents an example of important groups aged 65 to 75 years with a range of PSA and Gleason values. The table does not show data on groups $<65$ years of age since RP is cost effective regardless of PSA and Gleason. The model can calculate ICERs for those aged $>75$ years, but the table does not present these data since this age group exceeds the SPCG-4 inclusion criteria and should therefore be treated with greater caution. The Swedish National Prostate Cancer Register (NPCR) reports that in 2008 about $10 \%$ of the patients were aged $<60$ years, $37 \%$ were aged 60 to 69 years, $33 \%$ were aged 70 to 80 years, and about $20 \%$ were aged $>80$ years when diagnosed with prostate cancer (www.vinkcancer.se). The results from this study are relevant for approximately $60 \%$ of the prostate cancer patients as regards age, but the figure could be somewhat lower since not all patients have localized cancer. The probabilistic reasoning is based on data from a Nordic randomized trial. We used Swedish mortality data since it should be most applicable to Nordic patient groups, but the model can 
be adjusted with input of international data to have greater validity for other countries. The model can also be adjusted in the future as better data become available on current conditions.

The major strength of this analysis compared to earlier model analyses is that the data come from a randomized trial, presumably excluding selection bias. The long-term follow-up also strengthens the result. Long-term follow-up enhances data credibility since it enables researchers to identify long-term treatment effects. The study includes actual resource-use data, i.e. the cost data are not based on hypothetical assumptions as is often the case in other similar studies.

A limitation of the analytic method presented here is that it simulates from study data and extrapolates data to a lifetime horizon, instead of following up the patients until they die. The problem with the latter approach is that it is expensive and takes many years. Moreover, clinical guidelines and treatment methods could change during a prolonged follow-up period. This study has a long-term median follow-up of 10 to 12 years, and we have little reason to believe that a dramatic change in the data would occur after this period. A limitation concerning Gleason could affect the results of this study; specifically, the SPCG-4 included only patients with localized prostate cancer in curable ages $(<=75$ years), which leads to underrepresentation of Gleason 8-10.

Even if the data present good internal validity, their external validity could be discussed, e.g. perhaps the result is not fully applicable in different clinical contexts relative to the period of data collection. The first patient was enrolled in 1989, and treatment methods have improved since then. Moreover, new treatment modalities, e.g. robotic surgery, have been introduced and are now commonly used. However, this procedure is more expensive than RP, and its treatment effects compared to RP have not been fully investigated. Due to widespread PSA screening, more indolent tumors are being diagnosed, and concomitant overtreatment has become a major clinical and ethical dilemma. Active surveillance is increasingly used to 
counteract the problem of overtreatment. This may be taken into consideration in the decision-making process regarding treatments. The decision support model should be updated with active surveillance data when long-term data from randomized trials becomes available.

\section{Conclusions}

The cost-effectiveness ratio for RP varies with age, Gleason score, and PSA values. Assuming a threshold value of 200000 SEK per QALY gained, in patients aged $<=70$ years the treatment is always cost effective, except at age 70 , Gleason $0-4$, and PSA $<=10$. Using the same threshold value at age 75, Gleason 7-9 (regardless of PSA) and Gleason 5-6 (with PSA > 20) are cost effective. Hence, RP is not perceived to be cost effective in males aged 75 years having low Gleason and low PSA. Higher threshold values for patients with clinically localized prostate cancer could be discussed. 


\section{Acknowledgements}

The authors would like to thank Hans Garmo and Martin Henriksson for contributing to this article.

Declaration of interest: The authors report no conflicts of interest. The authors alone are responsible for the content and the writing of this paper. 


\section{References}

1. Kvale R, Auvinen A, Adami HO, Klint A, Hernes E, Moller B, et al. Interpreting trends in prostate cancer incidence and mortality in the five Nordic countries. J Natl Cancer I. 2007 Dec 19;99(24):1881-7.

2. Ung JO, Richie JP, Chen MH, Renshaw AA, D'Amico AV. Evolution of the presentation and pathologic and biochemical outcomes after radical prostatectomy for patients with clinically localized prostate cancer diagnosed during the PSA era. Urology. 2002 Sep;60(3):458-63.

3. Klotz L, Zhang LY, Lam A, Nam R, Mamedov A, Loblaw A. Clinical Results of LongTerm Follow-Up of a Large, Active Surveillance Cohort With Localized Prostate Cancer. J Clin Oncol. 2010 Jan 1;28(1):126-31.

4. Shortliffe EH. Medical informatics : computer applications in health care and biomedicine. 2nd ed. New York: Springer; 2001.

5. Griffin S, Claxton K, Hawkins N, Sculpher M. Probabilistic analysis and computationally expensive models: Necessary and required? Value Health. 2006 Jul-Aug;9(4):244-52.

6. Hummel S, Paisley S, Morgan A, Currie E, Brewer N. Clinical and cost-effectiveness of new and emerging technologies for early localised prostate cancer: a systematic review. Health Technol Assess. 2003;7(33):iii, ix-x, 1-157.

7. Calvert NW, Morgan AB, Catto JWF, Hamdy FC, Akehurst RL, Mouncey P, et al. Effectiveness and cost-effectiveness of prognostic markers in prostate cancer. Brit $\mathbf{J}$ Cancer. 2003 Jan 13;88(1):31-5.

8. Kattan MW, Cowen ME, Miles BJ. A decision analysis for treatment of clinically localized prostate cancer. J Gen Intern Med. 1997 May;12(5):299-305.

9. Holmberg L, Bill-Axelson A, Helgesen F, Salo JO, Folmerz P, Haggman M, et al. A randomized trial comparing radical prostatectomy with watchful waiting in early prostate cancer. New Engl J Med. 2002 Sep 12;347(11):781-9.

10. Bill-Axelson A, Holmberg L, Ruutu M, Haggman M, Andersson SO, Bratell S, et al. Radical prostatectomy versus watchful waiting in early prostate cancer. New Engl J Med. 2005 May 12;352(19):1977-84.

11. Bill-Axelson A, Holmberg L, Filen F, Ruutu M, Garmo H, Busch C, et al. Radical prostatectomy versus watchful waiting in localized prostate cancer: the Scandinavian Prostate Cancer Group-4 randomized trial. J Natl Cancer I. 2008 Aug 20;100(16):1144-54.

12. Bill-Axelson A, Holmberg L, Ruutu M, Garmo H, Stark JR, Busch C, et al. Radical prostatectomy versus watchful waiting in early prostate cancer. N Engl J Med. 2011 May 5;364(18):1708-17.

13. Johansson E, Bill-Axelson A, Holmberg L, Onelov E, Johansson JE, Steineck G, et al. Time, Symptom Burden, Androgen Deprivation, and Self-Assessed Quality of Life after Radical Prostatectomy or Watchful Waiting: The Randomized Scandinavian Prostate Cancer Group Study Number 4 (SPCG-4) Clinical Trial. Eur Urol. 2009 Feb;55(2):42232.

14. Steineck G, Helgesen F, Adolfsson J, Dickman PW, Johansson J, Norlen BJ, et al. Quality of life after radical prostatectomy or watchful waiting. New Engl J Med. 2002 Sep 12;347(11):790-6.

15. Andersson SO, Andrén O, Lyth J, Stark J, Henriksson M, Adami HO, et al. Managing localized prostate cancer by radical prostatectomy or watchful waiting: cost analysis of a randomized trial (SPCG-4). Scand J Urol Nephrol. In press.

16. Fleming C, Wasson JH, Albertsen PC, Barry MJ, Wennberg JE. A Decision-Analysis of Alternative Treatment Strategies for Clinically Localized Prostate-Cancer. Jama-J Am Med Assoc. 1993 May 26;269(20):2650-8. 
17. Armitage P, Berry G. Statistical methods in medical research. 3rd ed. Oxford ; Boston: Blackwell Scientific Publications; 1994.

18. Kirkwood BR, Sterne JAC. Essential medical statistics. 2nd ed. Malden, Mass.: Blackwell Science; 2003.

19. Briggs A, Sculpher M, Dawson J, Fitzpatrick R, Murray D, Malchau H. Modelling the cost-effectiveness of primary hip replacement: how cost-effective is the Spectron compared to the Charnley prosthesis? Technical report. York: Centre for health economics 2003. Report No.: CHE Technical Paper Series 28.

20. Nelder JA, Wedderburn RWM. Generalized Linear Models. Journal of the Royal Statistical Society Series A (General). 1972;135(3):370-84.

21. Olsson U. Generalized linear models : an applied approach. Lund: Studentlitteratur; 2002.

22. Claxton K. The irrelevance of inference: a decision-making approach to the stochastic evaluation of health care technologies. J Health Econ. 1999 Jun;18(3):341-64.

23. Claxton K, Sculpher M, Drummond M. A rational framework for decision making by the National Institute for Clinical Excellence (NICE). Lancet. 2002 Aug 31;360(9334):711-5.

\section{Electronic references:}

www.roc.se/prostata/spcg4.pdf

www.cran.r-project.org/web/packages/survival/survival.pdf

www.vinkcancer.se/Global/Diagnoser/prostatacancer/rapporter/prostataca_rapport_04_08.pdf 